\title{
BMJ Open Improving breast cancer outcomes for Aboriginal women: a mixed-methods study protocol
}

\author{
Vita Christie (D , , 1,2 MacKenzie Rice, ${ }^{3}$ Jocelyn Dracakis, ${ }^{3}$ Deb Green, ${ }^{4}$ \\ Janaki Amin (D) , ${ }^{1}$ Karen Littlejohn, ${ }^{5}$ Christopher Pyke, ${ }^{5}$ Debbie McCowen, ${ }^{6}$ \\ Kylie Gwynne ${ }^{1}$
}

To cite: Christie V, Rice M, Dracakis J, et al. Improving breast cancer outcomes for Aboriginal women: a mixed-methods study protocol. BMJ Open 2022;12:e048003. doi:10.1136/ bmjopen-2020-048003

- Prepublication history and additional supplemental material for this paper are available online. To view these files, please visit the journal online (http://dx.doi.org/10.1136/ bmjopen-2020-048003).

Received 16 December 2020 Accepted 05 January 2022

Check for updates

(c) Author(s) (or their employer(s)) 2022. Re-use permitted under CC BY-NC. No commercial re-use. See rights and permissions. Published by BMJ.

For numbered affiliations see end of article.

Correspondence to

Ms Vita Christie;

vita.christie@sydney.edu.au

\section{ABSTRACT}

Introduction Breast cancer is the most commonly diagnosed cancer affecting Australian women, and the second highest cause of cancer death in Australian women. While the incidence of breast cancer is lower in Aboriginal women than non-Aboriginal women, the mortality rate for Aboriginal women is higher, with Aboriginal women 1.2 times more likely to die from the disease. In New South Wales, Aboriginal women are 69\% more likely to die from their breast cancer than nonAboriginal women.

Co-design is a research method recognised to enhance collaboration between those doing the research and those impacted by the research; which when used with Aboriginal communities, ensures research and services are relevant, culturally competent and empowers communities as co-researchers. We report the development of a new protocol using co-design methods to improve breast cancer outcomes for Aboriginal women.

Methods and analysis Through a Community Mapping Project in 2018, we co-designed an iterative quantitative and qualitative study consisting of five phases. In Phase 1, we will establish a governance framework. In Phase 2, we will provide information to community members regarding the modified parts of the screening, diagnosis, treatment and follow-up processes and invite them to partake. In Phase 3, the research team will collect data on the outcomes of the modified processes and the outcomes for the women who have and have not participated. The data shall be analysed quantitatively and thematically in Phase 4 with Aboriginal community representatives and reported back to community. Lastly, in Phase 5, we evaluate the co-design process and adapt our protocol for use in partnership with other communities.

Ethics and dissemination This study has ethics approval of the Aboriginal Health and Medical Research Council ref:1525/19. The findings will be published in the literature, presented at conferences and short summaries will be issued via social media.

\section{INTRODUCTION}

Breast cancer is the most commonly diagnosed cancer in Australia, representing 28\% of all cancer incidence in women and the second highest number of deaths. ${ }^{1}$ Mortality due to breast cancer has declined significantly over

\section{Strengths and limitations of this study}

- This study will use mixed methods to identify milestones, enablers and barriers along the breast cancer prevention and treatment pathway for Aboriginal women.

- The collective impact co-design methodology strengthens community engagement by sharing power, knowledge and skill with community members and health services.

- Small study size limits the generalisability of the research findings.

- This study will not report the suboptimal treatment rates for Aboriginal women.

recent decades. This coincides with improved rates of early detection following introduction of national population-based mammography screening programmes ${ }^{2}$ and increasingly effective adjuvant therapies. Yet, Aboriginal and Torres Strait Islander women in Australia continue to face high mortality rates, despite an incidence of breast cancer on par with or less than non-Indigenous women. ${ }^{3}$ Between 1998 and 2013, there was no significant decrease in the Indigenous mortality rates for breast cancer in comparison to a significant decline for non-Indigenous women. ${ }^{3}$ When looking at the overall picture, tumour biology is shown to be only part of the picture in the disparity of survival outcomes; preventable causes relating to delayed diagnosis and treatment are also substantial contributors. ${ }^{4-6}$

Uptake of screening has increased over time among both non-Indigenous and Indigenous populations in Australia, however, a significant gap remains. Australia's national population-based screening programme BreastScreen offers free 2-yearly mammograms targeting women aged 50-74 years. In 2019, $41 \%$ of Aboriginal and Torres Strait Islander women in this age group participated compared with $54 \%$ of non-Indigenous 
women. ${ }^{7}$ As a consequence of the gap, Aboriginal and Torres Strait Islander women are more likely to be diagnosed at an advanced stage, experiencing worse disease outcomes and lower rates of survival. ${ }^{7-9}$

While age is the greatest risk factor for breast cancer, Aboriginal women are more likely to be younger than non-Aboriginal women at the time of diagnosis ${ }^{10}$ and receive more invasive surgical treatment compared with their non-Aboriginal counterparts. ${ }^{11}$ It is well established in the literature that this association likely contributes to difficulty in engaging Aboriginal women in regular and timely follow-up monitoring and care. ${ }^{12}$

There is a demonstrable need for improved screening, diagnostic and care pathways for Aboriginal women in Australia. ${ }^{13}$ The literature identifies numerous enabling factors and barriers which contribute to ease, or not, of access, timeliness and quality of care for Aboriginal women with regard to breast cancer screening and services. ${ }^{14-24}$

Overwhelmingly, barriers are related to the lack of cultural safety within health services. Aboriginal women are apprehensive about using services due to recent or historical experiences of racism, lack of culturally safe care and a deficit of resources featuring culturally appropriate educational and health promotion messages. Initiatives focused on resourcing community-led initiatives to raise awareness have been found to facilitate increased uptake and provide culturally safe care. ${ }^{15} 1619202225$ This care involved Aboriginal health workers and highlights the importance of primary healthcare following diagnosis. Furthermore, individuals were less likely to engage in services as a consequence of previous experiences or the experiences of women they knew with mammography and breast cancer. Feelings of shame or stigma were also cited, as was the impact of financial barriers and geographical remoteness, ${ }^{26}$ leading to travel and time away from family.

There is a body of evidence surrounding initiatives aimed at increasing breast screening among Aboriginal women which indicates that success is highest where there are partnerships with Aboriginal communitycontrolled organisations. ${ }^{24}$ These initiatives implemented culturally appropriate engagement strategies to address a range of social, cultural, personal and economic factors. An expanding evidence base supports the use of 'co-design' as a research methodology for the design, implementation and evaluation of successful, cost-effective and sustainable strengths-based solutions to health challenges among Aboriginal communities. ${ }^{27}$

Application of rigorous co-design methods to enhance breast cancer screening among Indigenous women internationally have been documented in the literature ${ }^{28-32}$ but not extensively. We also note in the literature that low participation in follow-up services, including antioestrogen treatment, ${ }^{33}$ however, there is a need for more translational research using co-design methodology that partners with Aboriginal women, their communities and community-controlled health organisations to develop comprehensive ecological framework for addressing barriers and improving women's engagement in screening as well as follow-up diagnostic and breast cancer care services.

The co-design approach of the research team follows the five stages of the collective impact model. These include (1) common agenda, (2) shared measurement, (3) mutually reinforcing activities, (4) continuous communication and (5) backbone support. This process provides a structured and shared process from conception, through to design, implementation and evaluation and increases the likelihood that health services will be used by Aboriginal people and that, as a result, health outcomes will improve. This approach has been validated with Aboriginal communities for translational health research. ${ }^{27}$

A preliminary mapping process co-designed with the communities in 2018 has informed this research. The Community Mapping Project (CMP) consisted of interviews with five Aboriginal breast cancer survivors from local communities, plus staff, clients and families of both from the local Aboriginal Health Service (AHS), the Advisory Committee of the AHS and other breast cancer care providers, including staff at the local oncology unit. The CMP explored experiences, priorities and preferences, particularly from the survivors' perspectives. It was a comprehensive mapping of existing screening, diagnosis, treatment and post-treatment service delivery, resulting in co-designed recommendations to adjust the existing services and improve the pathway for Aboriginal women in the area. A draft patient journey and proposed modifications was drafted and checked with some members of the local community including a local women's group and one on one interactions. It was then presented to the clinicians from the Foundation for Breast Cancer Care, who provided input to the clinical pathways.

An important aspect of co-design is engaging with communities at the point the research is being conceptualised. This is well prior to ethics approval. The Aboriginal Health and Medical Rsearch Council (AHMRC) requires letters of support from Aboriginal Community Controlled Health Services. The way we obtain these letters of support is by engaging in a process, in this case the CMP, to co-design the protocol. Once the protocol is approved by communities and then the AHMRC, we proceed to implementation of the research. It is not possible therefore to report the detailed discussions, interviews and process of the CMP beyond the output, shown in table 1 and figures 1-5 (flowchart with key), because ethics approval was obtained after the co-design process of the CMP was completed and interviewees were assured that identity would not be recorded and findings would not be published.

This study will use the information garnered through the CMP to develop and evaluate a culturally safe breast screening, diagnostic, treatment and follow-up pathway for Aboriginal women in selected regions. The findings of this work will inform policy and practice aimed at 


\begin{tabular}{|c|c|}
\hline \multicolumn{2}{|c|}{$\begin{array}{l}\text { Table } 1 \\
\text { Project }\end{array}$} \\
\hline & Key recommendations \\
\hline 1 & $\begin{array}{l}\text { Screening days for Aboriginal women, staffed by Aboriginal } \\
\text { women }\end{array}$ \\
\hline 2 & $\begin{array}{l}\text { An Aboriginal health worker present at screening van for agreed } \\
\text { periods of time (outside of screening days) }\end{array}$ \\
\hline 3 & Provide transport to and from screening van \\
\hline 4 & $\begin{array}{l}\text { Community spokespeople promoting screening and extra support } \\
\text { offered for diagnosis, treatment and follow-up care }\end{array}$ \\
\hline 5 & Directory booklets for women who are receiving treatment \\
\hline 6 & $\begin{array}{l}\text { Directory booklets for health services in order to ensure they are } \\
\text { equipped with up to date information for women }\end{array}$ \\
\hline 7 & $\begin{array}{l}\text { Culturally appropriate area in local oncology department to } \\
\text { increase comfort for Aboriginal patients }\end{array}$ \\
\hline 8 & GP management plans prior to treatment \\
\hline 9 & Follow-up care plans post-treatment \\
\hline 10 & $\begin{array}{l}\text { Follow-up phone call/visit from an Aboriginal health worker to } \\
\text { check in and work through options }\end{array}$ \\
\hline 11 & Local Aboriginal cancer support group formed \\
\hline
\end{tabular}

GP, General Practitioner.

reducing the rates of mortality of Aboriginal women from breast cancer.

\section{AIMS AND OBJECTIVES \\ Primary objective}

In one region in New South Wales (NSW) Australia, we will evaluate a co-designed pathway for timely and culturally safe screening diagnostic, treatment and posttreatment services for Aboriginal women.

\section{Aim}

Improve communication and cultural safety of breast cancer prevention and treatment services or Aboriginal women.

\section{Flow chart mapping the} screening, diagnosis, treatment
and post-treatment process


Figure 1 Key to flow chart.

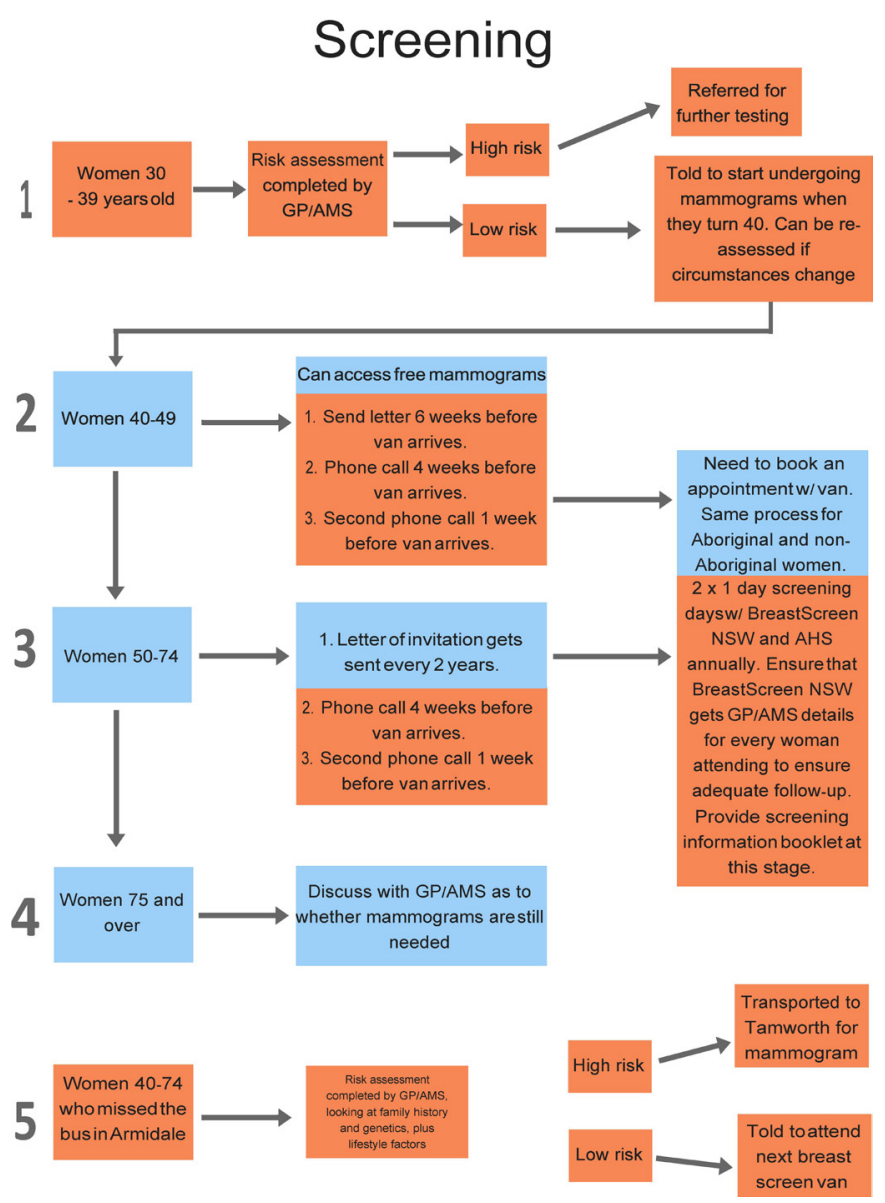

Figure 2 Screening services. AHS, Aboriginal Health Service; GP, General Practitioner; AMS, Aboriginal Medical Service; NSW, New South Wales.

\section{METHODS AND ANALYSIS \\ Co-design}

Co-design is a process of developing something with or alongside stakeholders or intended recipients or beneficiaries. ${ }^{27} 34$

The design of the study has been based on recommendations provided by the CMP from 2018. The CMP was a collaboration between the Foundation for Breast Cancer Care, the Poche Centre for Indigenous Health and Armajun Aboriginal Health Service. The CMP included consultation with Aboriginal survivors of breast cancer, Aboriginal women in the target age range for breast cancer prevention and early intervention, and Aboriginal health workers as well as local service providers in a regional and rural location situated in the Central North of New South Wales. The CMP informed this protocol. The development of the research question and outcome measures were informed by patients' and healthcare providers' priorities, experience and preferences.

The CMP guided the development of this iterative mixed methods study consisting of five main phases which makes adjustments to the present processes of screening, diagnosis, treatment and post-treatment follow-up to enhance cultural safety and promote engagement of Aboriginal women. The planned time period for data collection is 


\section{Diagnosis}

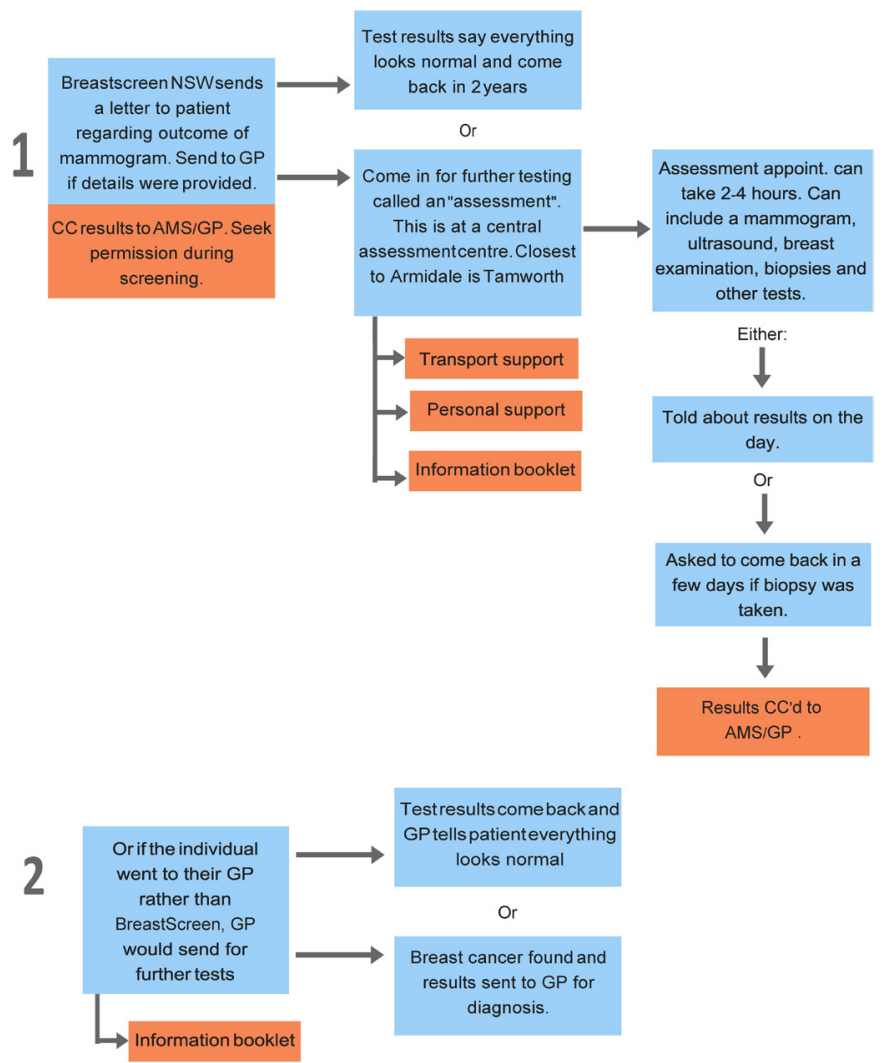

Figure 3 Diagnostic services. NSW, New South Wales; GP, General Practitioner; CC, Carbon Copy; AMS, Aboriginal Medical Service.

1 year: 2021-2022. The study commenced when ethics approval was granted in 2019 and will conclude 2022.

\section{Patient and public involvement}

Fundamental to the co-design model is patient and public involvement, with community organisations and members involved in the conception, design, implementation and evaluation of the study.

\section{Co-designed assessment and treatment pathway}

The current screening, diagnosis, treatment and posttreatment services respectively and describe the adjustments and additions created through the CMP are shown in figures $1-5$.

\section{Governance}

Through the CMP, it was determined how the local community wanted to be involved in the design, recruitment, implementation and evaluation of the study. Through the co-design process, the community was able to assess the burden of the research, including time and resource commitments, in order to inform a sustainable approach.

Local community members, breast cancer survivors and AHS staff will have continued input throughout the study as part of the iterative co-design process. An Aboriginal

\section{Treatment}

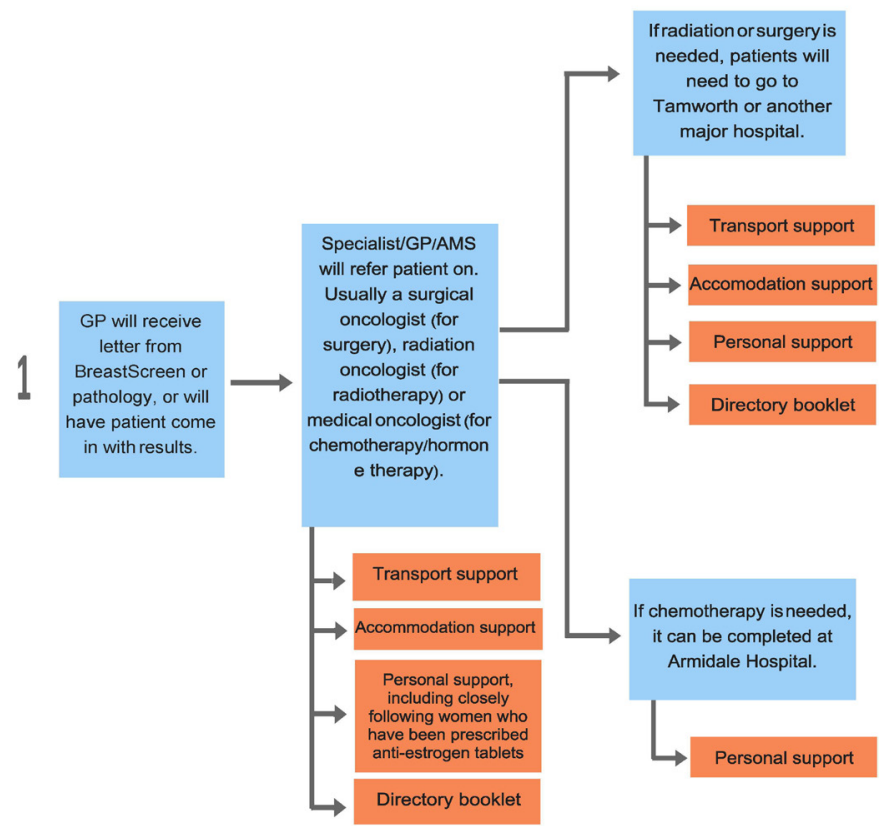

Figure 4 Treatment services. GP, General Practitioner; AMS, Aboriginal Medical Service.

Advisory Group will inform the study and its progress and AHS staff who self-nominate will be investigators on the study. The Aboriginal Advisory Group will meet every month and the Investigators' Group every 8 weeks. Data will be collected and owned and managed by the AHS and will be shared with Investigator group. Any publication will need to be approved by the Aboriginal Advisory Group and the AHMRC ethics committee. Members of the Investigators Group will continue to participate in community meetings regarding the progress and outcomes of the process, and community members will be given the option to receive a copy of the completed study and its outcomes and be invited to attend community meetings/workshops organised to disseminate the results of the study. The Aboriginal Advisory Group and AHS staff will be involved in designing the dissemination process.

\section{Ethics}

This project has ethics approval from the Aboriginal Health and Medical Research Council of NSW, Ref: $1525 / 19$.

\section{Design}

The study will use a mixed-methods design. Qualitative methods will include semi-structured, in-depth interviews and focus group discussions. Structured quantitative and qualitative questionnaires will be used to assess participant satisfaction, as well as collect information about demographics, patient journey and clinical data. 


\section{Post Treatment}

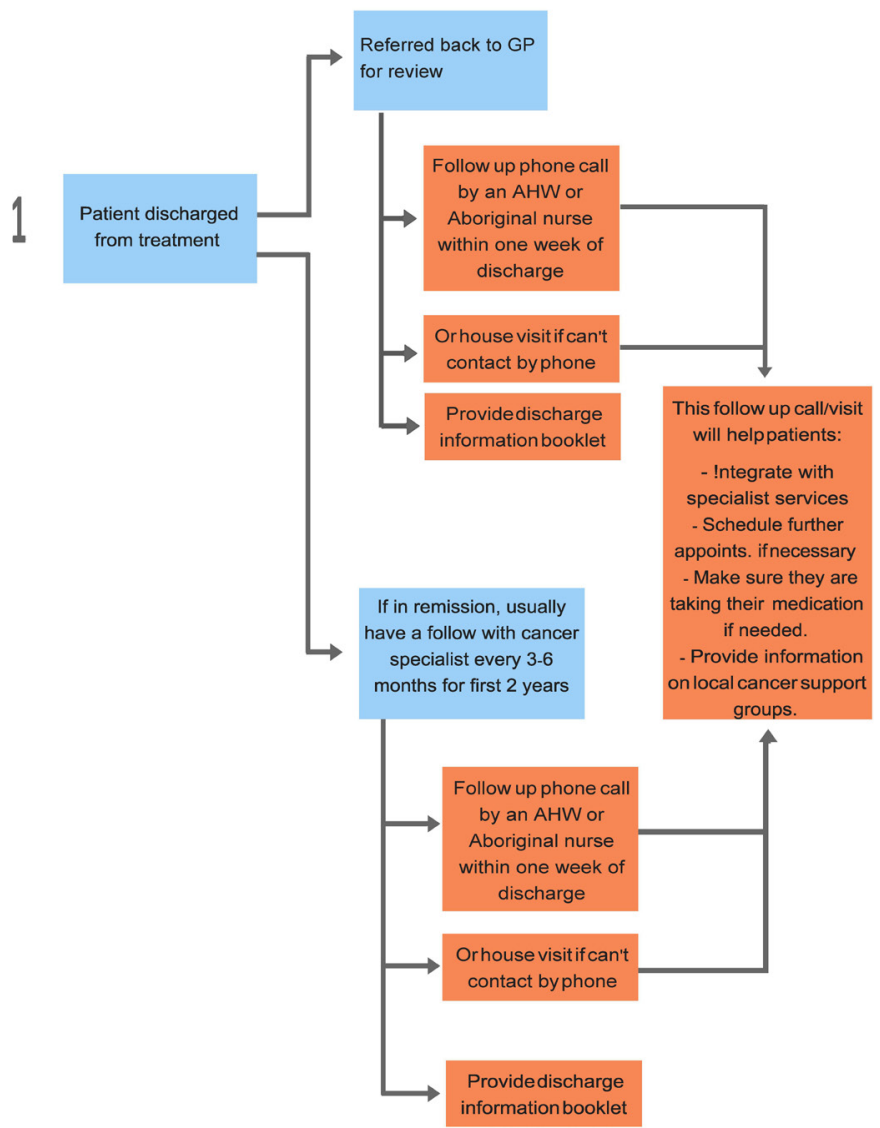

Figure 5 Post-treatment services. GP, General Practitioner; AHW, Aboriginal Health Worker.

\section{Target population}

The target population for this study is Aboriginal women living in the study region. The expected duration of this study is approximately 3 years, in which time the BreastScreen van will have visited these areas at least two times.

The area we are working with is a regional and rural location situated in the Central North of New South Wales. Seven per cent of the local population in the region identify as Aboriginal and/or Torres Strait Islander (ABS 2016 census data) which calculates as a possible sample size of 631 Aboriginal women above the age of 30 (according to AIHW 2018: 'For Indigenous Australians, five year relative survival was lowest in life stage 25-44 and increased with life stage. For non-Indigenous Australians, five year relative survival was similar for life stage 15-24, 25-44 and 45-64 and decreased in life stage 65 or older $\left.^{3}\right)$. Four specific study sites have been selected by convenience sampling on the basis of a pre-existing relationship with the local AHS which services the region. The communities expressed interest in working with the AHS in this capacity. The study will rely on voluntary sampling of individuals who fit the criteria of target population: Aboriginal women, $>30$ years and willing to receive care through the AHS.



Figure 6 Process of implementation.

\section{PHASES OF IMPLEMENTATION}

The process of implementation is shown in figure 6 .

\section{Phase 1: preparation}

The investigator team will work with BreastScreen NSW to gather the pre-intervention data to use as a baseline to measure quantitative outcomes. Data collected will include: retrospective data through the AHS related to participation as referral data is not available; number of women who attended the BreastScreen van in the region in 2018 and 2019 (2020 data has been affected by COVID-19 and therefore is not representative), and how many of these women identified as Aboriginal and/or Torres Strait Islander. The team will also work closely with the AHS (one of the investigator team is a staff member and second is the Chief Executive Officer of the AHS) in planning for a screening event, which is a fixed period of time where the van is reserved for Aboriginal women only and, additionally, staffing the van with an Aboriginal health worker for several extra days during its visit to the area. This work will be done in consultation with BreastScreen NSW.

The team will also work on development of resources for patrons of the local health services, including information on the different stages of the treatment journey and on the local support services for these different stages. This will include working with the local oncology unit to ensure that welcoming and culturally safe spaces are offered to Aboriginal women in the area.

\section{Phase 2: community information}

In our co-design process, 'community information' refers to both promoting the culturally safe screening events and providing relevant information to potential participants regarding the study. The local AHS staff and Investigator team member will reach out to communities in the region to inform them of the screening day event and presence of an Aboriginal health worker in the BreastScreen van in 20212022, and the inclusion of additional support services such as 
transport and child-minding. Outreach will include several forms of communication such as phone calls, emails, flyers and community visits, and will include written reminders 1 week out from the screening day date.

Screening and follow-up will be undertaken according to the procedures described in figures $1-5$. The AHS will offer risk assessments for women between 30 and 39 years who will be referred on for screening and MRI if they are found to meet high risk criteria. For women who are 40-49 years old, letters will be sent out 6 weeks prior to screening dates and then two follow-up calls will be placed 4 weeks prior and 1 week prior to the screening dates. For women who qualify for a reminder sent out by the government (50-74 years old), reminder phone calls will be placed 4 weeks prior and 1 week prior to the screening dates. For the women who are referred for further diagnosis, resources will be given to and support from the local Aboriginal health workers on next steps.

Additionally, there will be Aboriginal health workers acting as support staff if treatment is suggested, to inform affected women of the resources on offer and treatment pathways options. Women who partake in any part of the modified screening, diagnosis, treatment or follow-up processes will be invited to join the study but there will be no consequences for their treatment or support if they elect not to participate. The AHS will invite all women who have survived breast cancer and registered with the health service to participate in focus groups.

\section{Phase 3: data collection and outcome measurement}

All participants involved in the study will be recruited via the AHS. The AHS will contact each prospective participant personally and explain the study to them. They will explain the Participant Information Sheet and seek informed consent (PIS) (see online supplemental appendix 1). The PIS outlines what the study is about; who is carrying out the study; what the study involves; information about how they can withdraw from the study; information about the risks and benefits of the study and information on how to contact the researchers if needed.

All participants who complete the satisfaction questionnaire, participate in the in-depth interviews, or participate in the focus group discussions will need to sign a consent form. This consent form will allow the study to use the information provided and the participants will be advised the information they provide will be de-identified. The consent process will be managed by the AHS. Women participating in the in-depth interviews and focus group discussions will be advised that these will be recorded. These recordings will be transcribed, de-identified, and then destroyed.

All Aboriginal women in the community will be allowed to access any of the additional services/modifications being implemented, regardless of participation in the study. This will ensure equitable access to all services provided and will not negatively impact Aboriginal women who do not feel comfortable participating in the study.

The Aboriginal health worker will manage the consent process and consents will be held by the AHS. Consents will be gathered prior to participation in screening, in-depth interviews and focus groups.

The quantitative data (numbers of attendees who identify as Aboriginal and Torres Strait Islander) will be collected by the AHS at the culturally safe screening events and satisfaction questionnaires will be supplied after the event via the AHS. The qualitative data will be gathered during the in-depth interviews conducted by the AHS at a location acceptable to the participants between two and 4 weeks after the event so as to capture the experience when it is fresh in the minds of the participants. The focus groups will be organised by and held at the local AHS and will include a shared meal over which a series of questions will be asked (see online supplemental appendix 2_Questions for focus groups) and responses collected. The discussions will be audio recorded and transcribed with the consent of the participants.

Further qualitative data will be collected from women engaging in the diagnosis, treatment and follow-up processes via focus groups and interviews. This data aims to capture level of satisfaction with the modifications outlined in the flow charts. The modifications include, but are not limited to, increased communication and support from AHS and specialists around uptake of effective treatment options, access to information regarding treatment options and comprehensive information regarding support available during treatment and post-treatment timeframe.

The AHS will also follow-up with the participants to review their well-being after focus groups and interviews.

The study will quantitatively measure:

- The participation rates of Aboriginal women in breast cancer screening (the van will visit the region two times within the 12-month data collection phase of the study).

- The participation rates of Aboriginal women in timely and culturally safe diagnostic, treatment and posttreatment services (over a 1-year period).

- Satisfaction levels among Aboriginal women in the local community who go through the screening, diagnostic, treatment and post-treatment process (over a 1-year period).

- Breast cancer health literacy among Aboriginal women in the local community (over a 1-year period).

To determine the change in rates, study outcomes will be compared with the baseline participation of Aboriginal women in breast screening service, defined as the number of women who attended the BreastScreen van in the chosen area and the surrounding regions in the $2-3$ years preceding and how many of these identified as Aboriginal and/or Torres Strait Islander.

To establish baseline satisfaction levels all women will be asked about satisfaction for each of the four stages (screening, diagnosis, treatment and follow-up). For each stage, the subset of women who have previously accessed that stage will be asked if there is any change in their level of satisfaction. Questionnaires will also be completed by Aboriginal women who have not accessed the BreastScreen van previously, asking them why they have not. 
Qualitative and quantitative data analysis will be used to establish whether the additional adjustments implemented were successful in improving attendance and satisfaction levels in breast cancer outcomes among Aboriginal women in the region.

Participation rates will be measured during the screening day event and at the clinics which have an Aboriginal health worker present and will be compared with data ascertained from BreastScreen van during 2018-2019. Participation in diagnostic, treatment and post-treatment services will be tracked in the 6 months post screening event via the AHS, which will follow-up with the local treatment services and with the women who have received advice to go for further screening and treatment. This will entail a phone call and welfare check and further referrals and advice as indicated.

To establish whether there was an improvement in satisfaction levels regarding screening, services available to Aboriginal women questionnaires will be completed by those who accessed the screening van in 2021-2022, both during normal operation and the screening day event (see online supplemental appendix 3). Questionnaires and in depth interviews will also be completed by women who accessed diagnostic, treatment and/or posttreatment services for breast cancer in 2021-2022.

Semistructured interviews will be conducted to gather more in-depth, qualitative data with women who visited the BreastScreen van when it was running typically, those who visited during the screening day event and also those who knew about the event but chose not to attend. In addition, 2-hour focus groups will be conducted with $8-10$ women who attended while it was running typically and 8-10 women who attended the Screening day event.

The Australian Commission on Safety and Quality in Healthcare definition of health literacy is 'about how people understand information about health and healthcare, and how they apply that information to their lives, use it to make decisions and act on it. Health literacy is important because it shapes people's health and the safety and quality of healthcare'. ${ }^{35}$ The way this study will assess health literacy will be through increase in participation of services (quantitative data collection) and also a selfreported increase in understanding about breast cancer and the options for screening, diagnosis, treatment and follow-up care (via qualitative focus group discussion).

\section{Safety considerations}

Safety of the participants and anyone involved in the research is a priority of the study. Any adverse events during the study will be recorded and reported, and a follow-up of the event will be completed. The AHS will be available to assist and refer.

\section{Phase 4: data analysis and dissemination}

Data analysis plan

Quantitative data gathered through screening events and de-identified reports via the AHS primary healthcare software system and will be analysed through descriptive statistics.
For the qualitative data gathered through focus groups and in-depth interviews, we will perform an inductive thematic analysis using NVivo V.11 to organise participant's responses into key themes. Coding and thematic analysis of qualitative data will be carried out by two members of the study team and checked by an Aboriginal author who is also a member of the community, following best practices for enhancing validity in qualitative methods. ${ }^{36}$ The core research team will meet to review the findings and identify outstanding or representative quotes for future presentation of the results. Preliminary findings will be discussed with AHS and Aboriginal Advisory Group.

\section{Returning results to the community}

Results of the co-design study will be disseminated into the local Aboriginal community through community meetings, social media and printed research summaries (including Plain English summaries). We will work in collaboration with AHS to hold community meetings and information evenings, which both participants and non-participants of the study can attend. Community members will be encouraged to provide feedback and comments on the process.

Results will be published in peer reviewed journals and presented at professional conferences. The AHS participating in the study will be invited to contribute to these publications and presentations. We will acknowledge the sources of information and those who have contributed to the research through authorship and acknowledgement in resulting publications, meetings with community members and conference presentations. We will also acknowledge the cultural property rights of Aboriginal peoples in relation to knowledge, ideas, cultural expressions and cultural materials by including AHS representatives as research team members.

\section{Phase 5: adaptation}

In this phase, we will evaluate the co-design process to evaluate whether it can be adapted to similar health issues in Aboriginal communities elsewhere in Australia. We will do this by sharing the findings of the study widely across AHSs and social media and inviting AHSs to work with us to see if the protocol could be adapted with them for their community.

\section{Author affiliations}

${ }^{1}$ Faculty of Health and Human Sciences, Macquarie University, Sydney, New South Wales, Australia

${ }^{2}$ Poche Centre for Indigenous Health, The University of Sydney Faculty of Medicine and Health, Sydney, New South Wales, Australia

${ }^{3}$ The University of Sydney Faculty of Medicine and Health, Sydney, New South Wales, Australia

${ }^{4}$ Armajun Aboriginal Health Service, Armidale, New South Wales, Australia ${ }^{5}$ Foundation for Breast Cancer Care, Sydney, New South Wales, Australia

${ }^{6}$ Armajun Aboriginal Health Service, Inverell, New South Wales, Australia

\section{Twitter Kylie Gwynne @kyleis43}

Acknowledgements Foundation for Breast Cancer Care, Armajun Aboriginal Health Service, Poche Centre for Indigenous Health (Dr John Skinner and Associate Professor Lynette Riley). 
Collaborators Dr John Skinner, Assoc Prof Lynette Riley.

Contributors KG, DG, DM, MR, VC contributed to conception and design of the study. VC, KG, JD, DG contributed to planning. KG, CP, JA, DM, DG, VC contributed to governance and scientific advice. VC, KG, DG contributed to conduct and reporting. VC, MR, KG, JD contributed to drafting the Manuscript. VC, KG, JA, DG, KL, CP, DM, JD contributed to review and approval of the manuscript.

Funding This work was supported by the Foundation for Breast Cancer Care grant no. 20202716 (Macquarie University).

Competing interests None declared.

Patient consent for publication Not applicable.

Ethics approval This study involves human participants and was approved by Ethics Committee: Australian Health and Medical Research Ethics Committee, Reference 1525-19. Participants gave informed consent to participate in the study before taking part.

Provenance and peer review Not commissioned; externally peer reviewed.

Supplemental material This content has been supplied by the author(s). It has not been vetted by BMJ Publishing Group Limited (BMJ) and may not have been peer-reviewed. Any opinions or recommendations discussed are solely those of the author(s) and are not endorsed by BMJ. BMJ disclaims all liability and responsibility arising from any reliance placed on the content. Where the content includes any translated material, BMJ does not warrant the accuracy and reliability of the translations (including but not limited to local regulations, clinical guidelines, terminology, drug names and drug dosages), and is not responsible for any error and/or omissions arising from translation and adaptation or otherwise.

Open access This is an open access article distributed in accordance with the Creative Commons Attribution Non Commercial (CC BY-NC 4.0) license, which permits others to distribute, remix, adapt, build upon this work non-commercially, and license their derivative works on different terms, provided the original work is properly cited, appropriate credit is given, any changes made indicated, and the use is non-commercial. See: http://creativecommons.org/licenses/by-nc/4.0/.

\section{ORCID iDs}

Vita Christie http://orcid.org/0000-0003-3887-8305

Janaki Amin http://orcid.org/0000-0003-2161-9366

\section{REFERENCES}

1 Australian Institute of Health and Welfare. Cancer in Australia 2019 Canberra: AlHW, 2019.

2 Australian Institute of Health and Welfare. Breastscreen Australia monitoring report 2018. Canberra: AlHW, 2018.

3 Australian Institute of Health and Welfare. Cancer in Aboriginal \& Torres Strait Islander people of Australia. Canberra: AlHW, 2018.

4 Read DJ, Frentzas S, Ward L, et al. Do histopathological features of breast cancer in Australian Indigenous women explain the survival disparity? A two decade long study in the Northern Territory. Asia Pac J Clin Oncol 2020;16:348-55.

5 Redfern AD, Fong JJ, Spalding LJ. Abstract P5-04-18: unfavourable tumour stromal immune cell infiltrates but not circulating immune cell profiles in Aboriginal women with breast cancer. Cancer Res 2020;80.

6 Redfern AD, Spalding LJ, EYC L. Abstract P2-10-09: aggressive tumour biology contributes to poor breast cancer outcomes for Indigenous Australians. Cancer Res 2020;80.

7 Moore SP, Soerjomataram I, Green AC, et al. Breast cancer diagnosis, patterns of care and burden of disease in Queensland, Australia (1998-2004): does being Indigenous make a difference? Int J Public Health 2016:61:435-42.

8 Tapia KA, Garvey G, Mc Entee M, et al. Breast Cancer in Australian Indigenous Women: Incidence,Mortality, and Risk Factors. Asian Pac $J$ Cancer Prev 2017;18:873-84.

9 Roder D, Webster F, Zorbas $\mathrm{H}$, et al. Breast screening and breast cancer survival in Aboriginal and Torres Strait Islander women of Australia. Asian Pac J Cancer Prev 2012:13:147-55.

10 Dasgupta P, Baade PD, Youlden DR, et al. Variations in outcomes for Indigenous women with breast cancer in Australia: a systematic review. Eur J Cancer Care 2017;26:e12662.

11 Banham D, Roder D, Keefe D, et al. Disparities in breast screening, stage at diagnosis, cancer treatment and the subsequent risk of cancer death: a retrospective, matched cohort of Aboriginal and
non-Aboriginal women with breast cancer. BMC Health Serv Res 2019;19:387.

12 Christie V, Green D, Amin J, et al. What is the evidence globally for culturally safe strategies to improve breast cancer outcomes for Indigenous women in high income countries? A systematic review. Int J Environ Res Public Health 2021;18. doi:10.3390/ijerph18116073. [Epub ahead of print: 04 Jul 2021].

13 Thompson SC. Indigenous women and breast cancer. Med J Aust 2009;190:602.

14 Hedges S, Davidson M, Forrester S, et al. A breast screening Shawl to help aboriginal women feel more comfortable and culturally safe. $J$ Glob Oncol 2018:4:40s.

15 McMichael C, Kirk M, Manderson L, et al. Indigenous women's perceptions of breast cancer diagnosis and treatment in Queensland. Aust N Z J Public Health 2000;24:515-9.

16 Fox W, Powell M, Hyland V, et al, eds. Supportive care for women with breast cancer living in rural Australia. Cancer Forum. The Cancer Council Australia, 2017.

17 'Orchid' Breastscreen Queensland's New 4WD Digital Mobile Service 2010-08:34:39.

18 Campbell J, Kurnoth P. Well women making a diffence: evaluation report and program guide. Darwin: Women's Cancer Prevention Program, Department of Health and Community Services, Northern Territory Government, 2000.

19 Pilkington L, Haigh MM, Durey A, et al. Perspectives of Aboriginal women on participation in mammographic screening: a step towards improving services. BMC Public Health 2017;17:697.

20 Reath J, Carey M. Breast and cervical cancer in Indigenous women-overcoming barriers to early detection. Aust Fam Physician 2008;37:178.

21 Supramaniam R, Gibberd A, Dillon A, et al. Increasing rates of surgical treatment and preventing comorbidities may increase breast cancer survival for Aboriginal women. BMC Cancer 2014:14:163.

22 Byers L, Michell K, McCullough K. Awareness, acceptability and access to screening mammography for remote Aboriginal women. Health Promot J Austr 2018;29:366-7.

23 Byers L, Michell K, McCullough K, Awareness MK. Awareness, acceptability and access to screening mammography for remote Aboriginal women. Health Promot J Austr 2018;29:366-7.

24 Reath J, Carey M. Breast and cervical cancer in Indigenous women-overcoming barriers to early detection. Aust Fam Physician 2008;37:178-82.

25 Hedges S, Davidson M, Forrester S. A breast screening shawl to help aboriginal women feel more comfortable and culturally safe. Am J Clin Oncol 2018.

26 Valery PC, Bernardes CM, de Witt A, et al. Patterns of primary health care service use of Indigenous Australians diagnosed with cancer. Support Care Cancer 2020;28:317-27.

27 Gwynne K, Cairnduff A. Applying collective impact to Wicked problems in Aboriginal health. Metrop Univ 2017;28:115

28 Banner RO, DeCambra H, Enos R, et al. A breast and cervical cance project in a native Hawaiian community: Wai'anae cancer research project. Prev Med 1995;24:447-53.

29 Brown SR, Nuno T, Joshweseoma L, et al. Impact of a communitybased breast cancer screening program on Hopi women. Prev Med 2011; 52:390-3.

30 English KC, Fairbanks J, Finster CE, et al. A socioecological approach to improving mammography rates in a tribal community. Health Educ Behav 2008;35:396-409.

31 Ka'opua LSI, Park SH, Ward ME, et al. Testing the feasibility of a culturally tailored breast cancer screening intervention with native Hawaiian women in rural churches. Health Soc Work 2011;36:55-65.

32 Strickland CJ, Hillaire E. Conducting a feasibility study in women's health screening among women in a Pacific Northwest American Indian tribe. J Transcult Nurs 2016;27:42-8.

33 Redfern AD, Khan A, Spalding LJ. Abstract P2-10-16: breast cancer treatment patterns and outcomes for Australian women by Indigenous status and remoteness of residence. Cancer Research 2020;80.

34 Kania JHF. Essential Mindset shifts for collective impact. In: Stanford social innovation review, 2014.

35 Care $\mathrm{ACoSaQiH}$. Health literacy: taking action to improve safety and quality, 2014

36 Saldaña J. The coding manual for qualitative researchers / Johnny Saldaña. London: SAGE, 2009.

37 Hyett N, Kenny A, Dickson-Swift V. Methodology or method? A critical review of qualitative case study reports. Int J Qual Stud Health Well-being 2014;9:23606. 